\title{
Experimental Research on the Visual Characteristics of Urban Road Landscape Based on Driver's View
}

\author{
Li Shujian ${ }^{1, *}$ Wang Guan ${ }^{2}$ \\ ${ }^{1}$ ChangChun Institute of Technology, China \\ ${ }^{2}$ ChangChun Normal University, China
}

\begin{abstract}
The vision system is the main way for drivers to obtain information. The distribution of urban road landscape is one of the key factors that cause drivers to be visually irritating. This article selects typical urban road landscape environment sections to conduct driver visual search mode experiments, using quantitative evaluation and synthesis The qualitative evaluation method analyzes and compares the obtained eye dynamic data, explores the influencing factors of urban road landscape on the driver's visual characteristics and the law of visual change. Based on the experimental data, it is proposed that road landscape design should integrate greening design and guidance signs, Urban optimization strategies for building a coherent system of urban road landscapes.
\end{abstract}

\section{Introduction}

Urban road environment is a complex environmental system, which consists of people, cars, roads and environment ${ }^{[1]}$. Drivers are participants in outdoor activities, who are also the core part of urban road landscape environment research. Therefore, visual stimulation in the road landscape environment will have a certain impact on drivers' psychology, behavior and fatigue. ${ }^{[2]}$ Therefore, this paper analyzes the impact of urban road landscape environment on drivers' visual stimulation from the perspective of drivers, and proves its argument through the form of experiments, providing an important basis for ensuring drivers' safe driving and for creating a humane, comfortable and scientific urban road landscape environment.

\section{Problems existing in the road environment landscape under the driver's vision}

With the development of urbanization in recent years, the road landscape environment has been constantly enriched and changed, and its users and complex road environment have become a difficult problem in urban road landscape planning. Due to the motorization of people's travel, the complexity of road environment and the unsoundness of road system, the city has traffic congestion in some areas or in some periods of time, as well as poor traffic participation experience.

Drivers are one of the main groups involved in outdoor activities. In the road environment, drivers are mainly affected by traffic signs, signals, road greening, building environment, lighting and other facilities. There will be some limitations in planning only from the aspect of traffic or greening, which is also the crux of the problems existing in urban road landscape.

Generally speaking, it is mainly reflected in the following aspects:

In the driver's field of vision, the landscape elements are too complex. Too many indicators at the same location are not clear enough, which all reduce the accuracy and reaction time of the driver's identification, resulting in the confusion of information identification.

Because traffic indicator signs usually adopt a unified scale, when waiting at some wide intersections, the visual distance is far away. Therefore, the identifiability degree of information will be reduced, and the experience is poor.

The use of night landscape lights is not reasonable enough. There are too many landscape lights at night, which give too much light. It only emphasizes the beautification effect and ignores the stimulation of light to the driver's vision. Another example is the LED display of some street traffic signs. The light at night blurs the edge of the font and reduces the identifiability degree of the text. At the same time, the light emitted by the signs also stimulates drivers to different degrees. The recording and supplementary light at the intersection will disturb the passing vehicles and cause traffic accidents, which will have a great impact on the vision of drivers.

Road afforestation function is single, paying attention to beautification action only. Excessive green modelling or multifarious colour can distract the attention of driver. Thus, they should be used reasonably. In the design of urban road plant landscape, the function of separation and shelter should be strengthened. Furthermore, the function of identification and guidance should be enhanced to assist traffic passage.

\footnotetext{
* Corresponding author: 15135223@qq.com
} 
With the continuous development of urban construction, the increasing of road landscape elements has also led to significant changes in drivers' driving environment. Studying the visual perception of drivers in the road landscape environment is one of the important factors to construct a pluralistic, comprehensive and scientific landscape system.

\section{Experimental study on visual stimulation of road landscape to drivers}

In fact, good road traffic landscape environment is an essential content in traffic planning. It is necessary to provide a safe, efficient and scientific urban public environment system for traffic participants with its clear orientation, direction and information transmission. Through the following experiments, it can be shown that road landscape has a certain influence on drivers' vision, and the data can provide strong evidence for establishing a more scientific and human road environment landscape planning.

\subsection{Experiment section selection}

Two representative urban traffic arteries in Changchun were selected for the experimental sections, which are denoted as sections $\mathrm{A}$ and $\mathrm{B}$.

Section A is located in Yatai Street, with a total length of about $24 \mathrm{~km}$ and a width of about $46 \mathrm{~m}$. The road section includes two parts: expressway and urban road. The landscape elements along the road are complex, including expressway and urban road. Landscape elements mainly include vegetation, architecture, transportation facilities and so on.

Section B is located in South 3rd Ring Road (from Ecological Square to Weishan Road), with a total length of about $13 \mathrm{~km}$ and a width of about $48 \mathrm{~m}$. The road section is a general urban trunk road, with relatively single landscape elements and high vegetation greening rate.

The results of landscape heterogeneity evaluation data are shown in Table 1

Table1.Landscape heterogeneity index of two sections

\begin{tabular}{c|ccc}
\hline Road & $\begin{array}{c}\text { Richness } \\
\text { index }\end{array}$ & $\begin{array}{c}\text { Diversity } \\
\text { index }\end{array}$ & $\begin{array}{c}\text { Dominance } \\
\text { index }\end{array}$ \\
\hline $\mathrm{A}$ & 0.2873 & 0.5132 & 0.2871 \\
\hline $\mathrm{B}$ & 0.1736 & 0.2011 & 0.8327 \\
\hline
\end{tabular}

Through the analysis and calculation of Table 1, it can be seen that the landscape diversity index and richness index of Section $A$ are higher than that of Section B. However, its dominance index is lower than that of Section B, because the environmental landscape of Section A is diversified, leading to higher complexity and heterogeneity indexes. Section B is a typical urban road, with a simpler environment than Section A. It is mainly plant landscape, relatively low degree of heterogeneity.
Through the comparison of landscape heterogeneity between the two places, it can be seen that the difference is obvious and the data has certain representativeness, which can meet the experimental requirements.

\subsection{Preparation for experiment}

\subsubsection{Selection of experimental objects and instruments}

In order to ensure the quality of the experiment, 6 drivers of different genders and occupations were selected for the experiment, with their driving years ranging from 5 to 30 years and ages ranging from 29 to 53 years, as shown in Table 2.

Table 2 Driver details

\begin{tabular}{c|ccccc}
\hline Number & gender & age & $\begin{array}{c}\text { Driving } \\
\text { age }\end{array}$ & Occupation & Familiarity \\
\hline 1 & male & 53 & 30 & Driver & yes \\
2 & male & 45 & 15 & Staff & no \\
3 & male & 38 & 10 & Businessm & no \\
& & & & an & \\
4 & female & 42 & 8 & Teracher & no \\
5 & female & 29 & 5 & Student & no \\
\hline $\mathbf{6}$ & female & 32 & 5 & Staff & no \\
\hline
\end{tabular}

\subsubsection{Selection of experimental vehicles}

Experimental vehicles should have good handling and driving vision. Self-owned vehicles of testers, such as cars and SUVs, represent the driving status of most people on the road.

\subsubsection{Measuring instrument}

In order to make the data accurate, IviewXHED eye tracker with high reliability and high performance is adopted. The position of the eyeball and pupil, the position of gaze, and the area of interest when the driver observes the target are collected to obtain visual characteristic index data. The collected information data can meet the experimental requirements.

\subsubsection{Visual stimulation index selection}

In the process of driving, drivers search for information visually and complete the process of judgment and response [3]. The behaviors of saccades, fixation and blinking of drivers were used as indicators to measure visual stimulation. Quantitative evaluation and comprehensive qualitative evaluation were used to select visual stimulation indicators. The quantitative method is the minimum mean square deviation method.

The calculation formula of the minimum mean square deviation is as follows: 


$$
s_{i}=\left[\frac{1}{n} \sum_{i=1}^{n}\left(x_{i}-\bar{x}_{i}\right)^{2}\right]^{\frac{1}{2}}
$$

In Formula (1), $\mathrm{Si}$ represents the mean square deviation of samples of evaluation indexes for different evaluation objects.

$$
\bar{x}_{i}=\frac{1}{n} \sum_{i=1}^{n} x_{i}
$$

In Formula (2), $\bar{x}_{i}$ expresses the sample mean values of evaluation indexes for different evaluation objects.

If there is $\mathrm{k} 0(1 \leq \mathrm{k} 0 \leq \mathrm{n}) \quad S_{k 0} \approx \min _{1 \leq i \leq n}\left\{s_{i}\right\}$ and $\mathrm{Sk} 0 \approx 0$, then the evaluation index $\mathrm{xk} 0$ corresponding to $\mathrm{Sk} 0$ will be filtered out.

S1blink response, S2 blink time, S3 fixation frequency, S4 fixation time, S5 pupil diameter, S6 saccade times, S7 saccade time, S8 saccade amplitude, S9 saccade velocity and S10 saccade peak acceleration ${ }^{[4]}$ were successively used as experimental description objects. The data obtained were shown in Table 3 by calculating the minimum mean square deviation.

Table3 Calculation results of minimum mean square deviation

\begin{tabular}{|c|c|c|c|c|c|c|c|c|c|c|}
\hline \multicolumn{11}{|c|}{ Evaluation Index Mean } \\
\hline 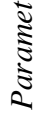 & $\bar{x}$ & $x^{2}$ & $\ddot{x}$ & $\star^{ \pm}$ & $\approx$ & 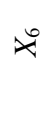 & 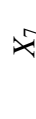 & $\infty$ & $x$ & $\stackrel{ْ}{\star}$ \\
\hline 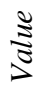 & $\vec{m}$ & $\begin{array}{l}\bar{\sim} \\
\tilde{m} \\
\stackrel{v}{ }\end{array}$ & $\vec{r}$ & $\begin{array}{l}\hat{\sigma} \\
\dot{\infty} \\
\dot{\sigma}\end{array}$ & $\frac{\check{\sigma}}{\dot{m}}$ & $\stackrel{n}{n}$ & $\begin{array}{l}\frac{3}{\gamma} \\
\text { f }\end{array}$ & $\underset{\mathrm{O}}{\tilde{I}}$ & $\frac{n}{\stackrel{\sim}{*}}$ & 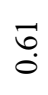 \\
\hline
\end{tabular}
of different indexes

\begin{tabular}{|c|c|c|c|c|c|c|c|c|c|c|}
\hline \multicolumn{11}{|c|}{ Minimum variance } \\
\hline 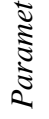 & $\overline{5}$ & బ & $\tilde{n}$ & பे & 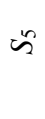 & $\omega^{\circ}$ & is & $\varkappa^{\infty}$ & ڤे & के \\
\hline$\frac{\Xi}{\Xi}$ & $\frac{\infty}{0}$ & $\begin{array}{l}\text { ô. } \\
\text { ते }\end{array}$ & $\begin{array}{l}\text { oे } \\
\stackrel{0}{0}\end{array}$ & $\begin{array}{l}\text { ָे } \\
\infty \\
\stackrel{\leftrightarrow}{2}\end{array}$ & 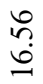 & $\stackrel{\infty}{\stackrel{\infty}{0}}$ & $=$ & $\begin{array}{l}\stackrel{0}{n} \\
\sim\end{array}$ & $\begin{array}{l}\stackrel{a}{0} \\
\stackrel{0}{0}\end{array}$ & $\stackrel{\circ}{\circ}$ \\
\hline
\end{tabular}

According to the data in table 3, the values of S1, S3, S6, S7, S9 and S10 tend to be close to 0, which have little influence on their fixation degree and cannot be used as evaluation indexes. The indicators of blink time, fixation time and pupil diameter and saccade amplitude have obvious changes, which can be used as evaluation indexes for analysis.

\subsection{Experimental results and analysis}

\subsubsection{Section A visual stimulation experiment results}

By collecting the information of blink time, fixation time and saccade amplitude of 6 drivers in Section A, the average value of various data was taken as the experimental result, and the corresponding curve was drawn according to the data intercepted at 10s, as shown in Figure 1, Figure 2 and Figure 3.

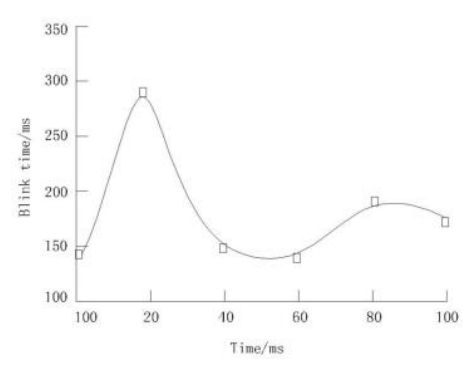

Fig. 1 Landscape on driver' s blink time

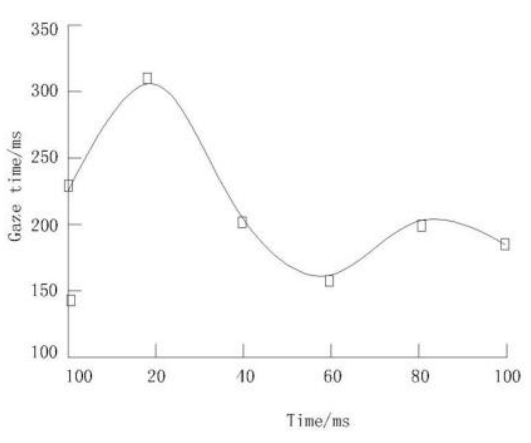

Fig. 2 Landscape on driver's gaze time

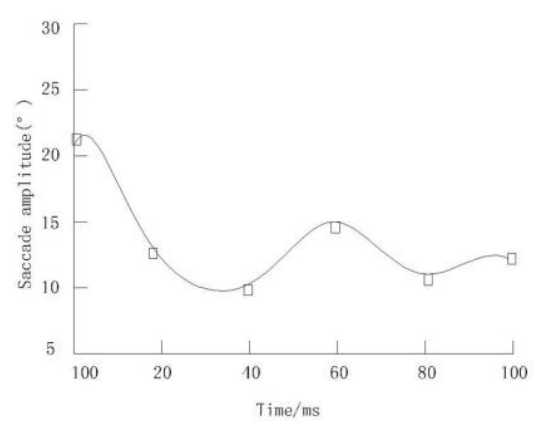

Fig. 3 Landscape on driver' saccade amplitude

From Figure 1 to Figure 3, it can be seen that blink response of drivers in the first $30 \mathrm{~s}$ of Section A significantly increased, with a large change range. The fixation time increased first and then decreased. There was a peak value especially when the driving time was 20 s. After 30 s, drivers gradually adapted to Section A, and the landscape saccade amplitude decreases.

Due to the complicated environment and landscape of Section A, drivers need to quickly acquire a large amount of information during driving. Especially at traffic junctions, the pupil diameter and fixation time of drivers increase, while the blink time and saccades decrease. Drivers are more focused and the data is more obvious. 


\subsubsection{Section B visual stimulation experiment results}

The data and methods of Section A are the same. The data curves of Section B are shown in Figure 4 to Figure 6.

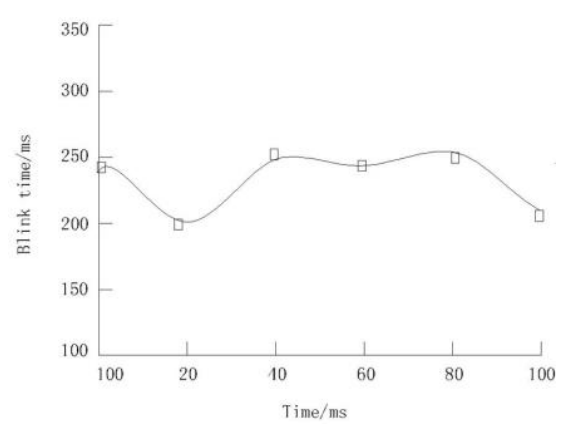

Fig. 4 Landscape on driver' s blink time

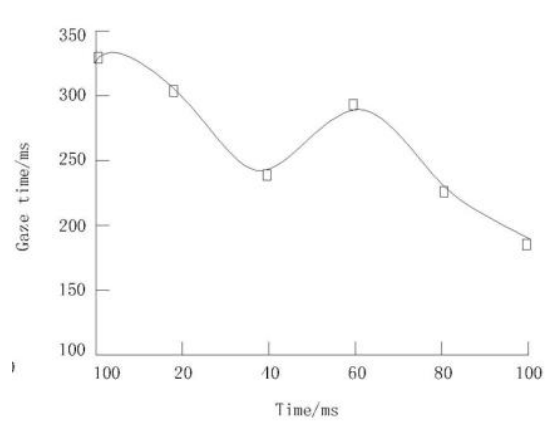

Fig. 5 Landscape on driver's gaze time

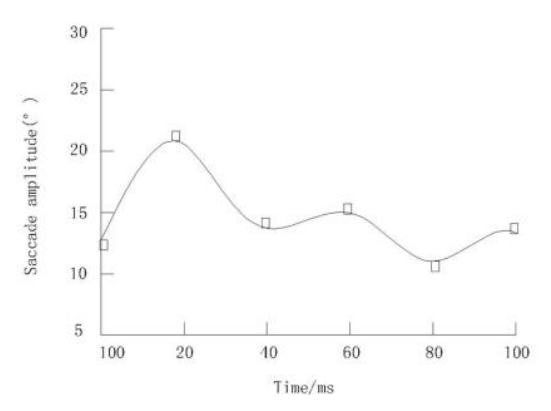

Fig. 6Landscape on driver' saccade amplitude

From Figure 4 to Figure 6, it can be seen that the blink time and fixation time of the driver in the first 30 s period change regularly. The pupil diameter fluctuates stably. However, in the first 30s, the saccade amplitude decreases from high and then increases again, with a large change range. This is because the landscape distribution of Section B is relatively monotonous, and drivers adjust faster in environments. During the period of 30-60s, the value of blink time decreased, and the variation of saccade amplitude and fixation time was relatively small, because the road section had a single environment, the driver was relatively relaxed, and the fluctuation range of each indicator was relatively small. The data shows that after 60 seconds, the fixation time value significantly decreased, the blink time significantly increased, and the saccade amplitude significantly decreased, indicating that the driver was prone to slip and fatigue when driving in the monotonous landscape distribution. Through self-adjustment, all indicators returned to normal.

\subsubsection{Statistical analysis of the influence of landscape on drivers' visual stimulation}

The visual stimulation indexes of Section A and Section $\mathrm{B}$ were compared to obtain the mean value, standard deviation and change rate of different indexes. The comparison results are shown in Table 4 to Table 6 .

Table4 Comparison of blink time statistics of two sections

\begin{tabular}{c|ccc}
\hline Road & Mean/ms & $\begin{array}{c}\text { Standard } \\
\text { deviation/ms }\end{array}$ & $\begin{array}{c}\text { Rate of } \\
\text { change/\% }\end{array}$ \\
\hline A & 223.31 & 43.42 & 15.31 \\
\hline B & 247.02 & 43.38 & 14.62 \\
\hline
\end{tabular}

Table5 Comparison of gaze time statistics of two sections

\begin{tabular}{c|ccc}
\hline Road & Mean/ms & $\begin{array}{c}\text { Standard } \\
\text { deviation/ms }\end{array}$ & $\begin{array}{c}\text { Rate of } \\
\text { change/\% }\end{array}$ \\
\hline $\mathrm{A}$ & 227.52 & 53.02 & 16.25 \\
\hline $\mathrm{B}$ & 304.61 & 47.81 & 14.53 \\
\hline
\end{tabular}

Table6 Comparison of scanning amplitude of two sections

\begin{tabular}{c|ccc}
\hline Road & Mean/ms & $\begin{array}{c}\text { Standard } \\
\text { deviation/ms }\end{array}$ & $\begin{array}{c}\text { Rate of } \\
\text { change/\% }\end{array}$ \\
\hline $\mathrm{A}$ & 15.51 & 2.87 & 17.05 \\
\hline $\mathrm{B}$ & 13.43 & 3.27 & 21.88 \\
\hline
\end{tabular}

According to the analysis of data in Table 4 to Table 6 , it can be seen that the driver's blink time and fixation time in Section A are lower than that in Section B, because the landscape environment of Section A is complex and the driver needs to obtain more information. However the landscape environment of Section B is simple and less information. The driver's eyes are focused and the saccade amplitude is small.

Through the study on the visual stimulation of urban road landscape distribution to drivers, it is proved that complex road landscape environment can reduce drivers' responsiveness. The more significant landscape elements can attract drivers' attention, the more closed visual field is conducive to concentration, and the lack of changing landscape environment will blunt drivers' attention. In addition, through interviews with experimental subjects, it is known that improper lighting setting at night also has strong visual stimulation on drivers. The above data can provide corresponding argument support for road landscape optimization.

\section{The optimization strategy of urban road landscape}

Based on the above experimental results, urban road landscape design should fully pay attention to drivers' visual factors. While meeting the basic behavioral needs 
of urban residents, we should ensure traffic safety, guide the traffic flow in and out quickly, improve traffic efficiency and safety, and build a road landscape environment with scientific, perfect and efficient functions. Therefore, the following urban road landscape optimization strategies are proposed.

\subsection{Safety first principle}

In order to pursue beautification or functionality, the traditional road landscape design often neglects the stimulating effect on drivers' vision, causing hidden danger of safety. Perfect and reasonable urban road landscape should adhere to the principle of safety first. The primary premise of traffic is to ensure the safe and fast passage of vehicles and pedestrians. Therefore, the road landscape design should consider the traffic characteristics and safety needs of the section, especially the special section. For example, the plant landscape at the traffic intersection should ensure that the vision of the driver is not blocked. Therefore, lawn and flowers can be planted. In addition, solitary planting of trees with higher branching points can be used as the characteristic marker to attract the attention of the drivers. Space belt greening design should prevent pedestrian crossing. So tall trees and coniferous species are good choices.

\subsection{Simplification and optimization}

The special shape, color and volume of the landscape can be optimized to define the boundary of the driver's vision and shape a good visual corridor. The unreasonable and unclear information marks are removed, and the unnecessary factors affecting the driver's line of sight are weakened and blocked by means of greening. The position size and brightness of the signal light should be optimized to enhance the identifiability degree. The night landscape lighting environment should be optimized, too.

\subsection{Emphasis on identifiability}

In the landscape design of road environment, we should base on the expression, transmission and acceptance of information. There are two things that can be done to enhance the identifiability degree of the environment. One is to strengthen the identifiability of characteristic markers, such as highlighting landmark buildings or structures, so as to facilitate identification. The other is to emphasize the identifiability degree of landscape in intersection and road node through the design of plants and sketches, so that drivers can make judgment in advance during driving.

\subsection{Integration of greening design and guiding sign design}

Greening is an important part of urban road landscape. Because of its beautification function, positive psychological stimulation is formed through visual stimulation to the driver. If the serialized space with clear structure and clear functional context can be created in the urban road landscape design, the road landscape environment will present orderly and standardized configuration mode, and the green landscape will become a beneficial supplement to the traditional traffic guidance system.

\subsection{Construction of consistency and system}

At present, the domestic urban road landscape is often lack of system. Its function is relatively isolated and scattered, reducing the identification, and the effective visual stimulation for drivers is relatively weak. According to the nature, function and surrounding environment of intersections, a unified landscape configuration method is applied in the same type of roads to form a clear and vivid urban landscape character. It not only helps drivers to predict the road situation ahead by identifying the road landscape type, but also helps to enhance the public's perception of the landscape environment and shape the image and charm of urban landscape.

\section{Conclusion}

The research shows that the road landscape has a certain influence on the vision of drivers. That is, the complex road landscape environment will reduce the driver's responsiveness. The more significant landscape elements can attract the driver's attention. The more closed visual field is conducive to concentration. The lack of changes in the landscape environment will blunt the driver's attention. In addition, through interviews with experimental subjects, it is known that improper lighting setting at night also has strong visual stimulation on drivers.

Based on the experimental results, this paper puts forward the optimization strategy of urban road landscape.So we should adhere to the principle of safety first, advocate the simplification and optimization design of urban road landscape, emphasize the identifiability of urban road landscape design, integrate the green design and guiding sign, and construct the continuity and systematization of urban road landscape.

\section{Acknowledgement}

Thanks to the school's scientific research team for their technical support in the process of forming this article, thanks to the careful guidance of the leaders, thanks to the enthusiastic participation of the volunteers participating in the experiment, and thanks to the academic achievements in the related fields, which provided a good academic for the research of this article basis. 


\section{References}

1. Kang Ning, Xiu Meiling. Effects of visual characteristics for different types of plant community on human psychology. Forestry University,2017;32(1):315-320

2. Qiao Jiungang, Zhou Tong, Zhou Ronggui. Study on road landscape based on the driver's heart physiological changes. Journal of Wuhan University of Technology,2015; 37(6):50-53

3. $\mathrm{He} \mathrm{Yu}$, Wang Biao, Gu Shining, et al. Vision system for autonomous landing of a micro our $r$ otor UAV. Computer Measurement

\& Control.2015;23(5):1682-1685

4. Schrank S J, Guy C S, Whiles M R, et al. Influence of instream and landscape-level factors on the distribution of topeka shiners notropis topeka in kansas streams. Copeia,2016;20(2):413-421 